\title{
Investigation of Microbubble Detection Methods for Super-resolution Imaging of Microvasculature
}

\author{
J. Brown, K. Christensen-Jeffries, S. Harput, G. Zhang, J. Zhu, C. Dunsby, M-X. Tang, and R. J. Eckersley.
}

\begin{abstract}
Ultrasound super-resolution techniques use the response of microbubble contrast agents (MBs) to visualize the microvasculature. Techniques that localize isolated bubble signals first require detection algorithms to separate the $M B$ and tissue responses. This work explores the three main MB detection techniques for super-resolution of microvasculature. Pulse inversion (PI), differential imaging (DI) and singular value decomposition (SVD) filtering were compared in terms of the localization accuracy, precision and contrast to tissue ratio (CTR).

MB responses were simulated based on the properties of Sonovue ${ }^{\mathrm{TM}}$ and using the Marmottant model. Non-linear propagation through tissue was modelled using the $k$-Wave software package.

For the parameters studied, the results show that PI is most appropriate for low frequency applications, but also most dependent on transducer bandwidth. SVD is preferable for high frequency acquisition where localization precision on the order of a few microns is possible. PI is largely independent of flow direction and speed compared to SVD and DI, so is appropriate for visualizing the slowest flows and tortuous vasculature. SVD is unsuitable for stationary MBs and can introduce a localization error on the order of hundreds of microns over the speed range 0 $2 \mathrm{~mm} / \mathrm{s}$ and flow directions from lateral (parallel to probe) to axial (perpendicular to probe). DI is only suitable for flow rates $>0.5$ $\mathbf{m m} / \mathbf{s}$ or as flow becomes more axial.

Overall, this study develops a MB and tissue non-linear simulation platform to improve understanding of how different MB detection techniques can impact the super-resolution process and explores some of the factors influencing the suitability of each.
\end{abstract}

Index Terms - Detection methods, super-localization, superresolution imaging, microbubbles.

\section{INTRODUCTION}

$\mathrm{T}_{\mathrm{n}}^{\mathrm{n}}$ HERE has been an interest in using ultrasound to visualize microvasculature for many years [1]. However, achieving the required micron resolution has proved challenging. Simply working at high transmit frequencies $(>20 \mathrm{MHz})$ can

Manuscript received (); This work was supported in part by the EPSRC under Grant EP/N015487/1 and Grant EP/N014855/1, in part by the King's College London and Imperial College London EPSRC Centre for Doctoral Training in Medical Imaging under Grant EP/L015226/1, in part by the Wellcome EPSRC Centre for Medical Engineering at King's College London under Grant WT $203148 / \mathrm{Z} / 16 / \mathrm{Z}$ and in part by the Department of Health through the National Institute for Health Research Comprehensive Biomedical Research Center Award to Guy's and St Thomas' NHS Foundation Trust in partnership with the King's College London and King's College Hospital NHS Foundation Trust. (Robert J. Eckersley, Christopher Dunsby, and Meng-Xing Tang contributed equally to this work.) (Corresponding author: Jemma Brown.)

J. Brown, K. Christensen-Jeffries, and R. J. Eckersley are with the Department of Biomedical Engineering, School of Biomedical Engineering and decrease the point spread function sufficiently to visualize superficial microvasculature. For example, Goertz et al. (2000) used a $50 \mathrm{MHz}$ center frequency to detect flow in a mouse ear vessel that was below $20 \mu \mathrm{m}$ in diameter [2]. However, attenuation in tissue increases at these frequencies, limiting this technique to superficial imaging. The contrast of blood vessels can also be increased by introducing microbubble contrast agents [3]. MBs are blood pool agents which consist of gas cores encapsulated in a shell which is engineered for stability [4]. Acoustic angiography is a contrast technique which uses low frequency transmission and MBs but only receives the high frequency signal components [5-7]. Gessner et al., (2013) showed that resolutions of $\approx 150 \mu \mathrm{m}$ axially and $\approx 200 \mu \mathrm{m}$ laterally at depths of $5 \mathrm{~mm}$ can be achieved [5]. However, resolutions achieved using these techniques are still limited by the fundamental diffraction limit.

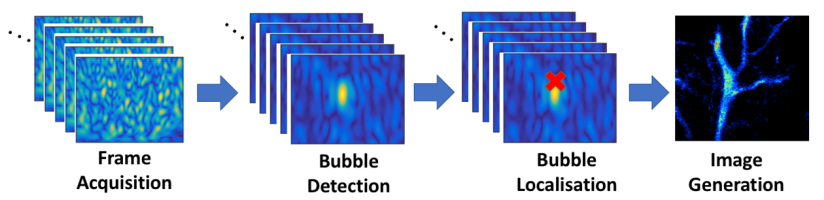

Fig. 1. Flow diagram of super-resolution process. Following the initial image acquisition, the MB detection algorithm separates the MB and tissue signal. Then isolated MBs can then be localized and the final image generated by combining localizations.

Super-resolution ultrasound imaging has the potential to visualize microvasculature noninvasively at depth [8-12]. Currently, super-resolution imaging methods using MBs can be divided into two streams; those using isolated MB signals [814] and statistical approaches [15, 16]. Given favorable conditions such as low noise and tissue attenuation, it is possible for MBs to scatter strongly enough for individual MBs to be identified and localized. Over time these localizations can be built up to visualize the vasculature. Thus, the achievable

Imaging Sciences, King's College London, London SE1 7EH, U.K. (e-mail: jemma.brown@kcl.ac.uk; kirsten.christensen- jeffries@kcl.ac.uk; robert.eckersley@kcl.ac.uk).

S. Harput, G. Zhang, J. Zhu, and M.-X. Tang are with the Ultrasound Laboratory for Imaging and Sensing Group, Department of Bioengineering, Imperial College London, London SW7 2AZ, U.K. (e-mail: s.harput@imperial.ac.uk; j.zhu16@imperial.ac.uk; mengxing.tang@imperial.ac.uk).

C. Dunsby is with the Department of Physics and the Centre for Pathology, Imperial College London, London SW7 2AZ, U.K. (e-mail: christopher.dunsby@imperial.ac.uk). 
resolution is no longer restricted to that of the diffraction limit but the precision with which the MB can be localized. The statistical methods apply higher order statistics to signal densities that are too concentrated for single bubble localization. Statistical approaches can achieve very short scan times of $<1 \mathrm{~s}$ but may show only a slight improvement (reducing the point spread function by up to $50 \%$ ) in spatial resolution [15] compared to isolated MB techniques performed at sufficiently low contrast agent concentrations. Previous demonstrations of super-resolution using isolated MBs have shown improvements in spatial resolution of several times smaller than the diffraction limited point spread function (PSF) $[8,10-12,17]$. As accurate super-resolution using isolated MB signals requires a reliable understanding of how the MB PSFs relate to MB position it is especially important to understand how any variation of the MB signals introduced by the detection techniques may cause localization errors. Thus, this work focuses on the isolated MB approach.

This work compares methods of extracting the MB response from the surrounding tissue signal. The three detection methods shown in Figure 2 have been investigated. Pulse inversion isolates the non-linear components of the received signal [18]. The second harmonic content of MBs allows this non-linear method to extract isolated microbubble signals from linear background [8]. Linear methods that detect microbubbles using movement due to flow have also been used. Desailly et al. (2013) introduces a differential imaging (DI) technique, where successive frames are subtracted and the resulting signal localized [10]. Here movement, destruction or dissolution of individual MBs within a cloud can generate a difference in signal between the frames which is revealed by the subtraction. Errico et al., (2015), developed the use of variation between frames through the application of a SVD filter which separates bubble and tissue signal based on the spatial and temporal characteristics of their acoustic response [12].

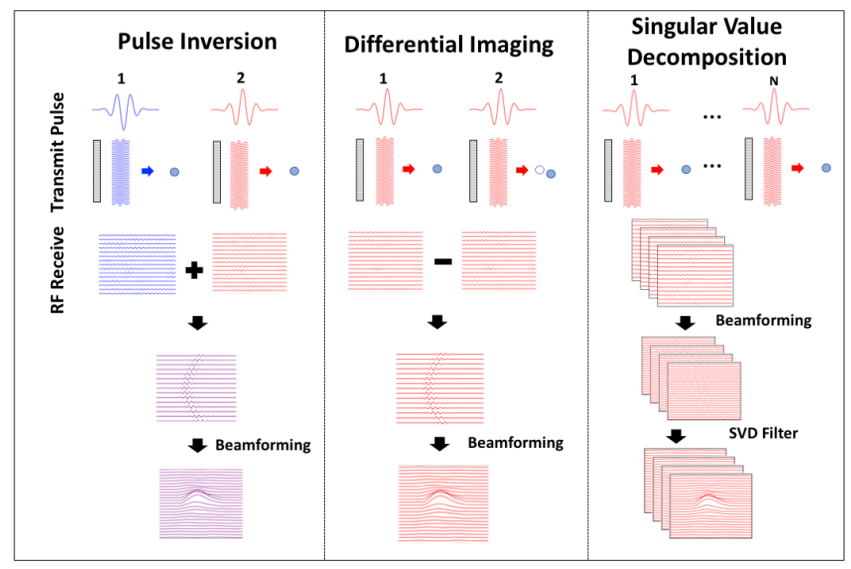

Fig. 2. Schematic of MB detection methods. Pulse inversion and differential imaging both combine two frames of data. Where red is associated with a positive pulse and blue is 180 degrees out of phase. SVD requires a stack of $\mathrm{N}$ images. In this work the SVD filter is applied to beamformed RF data before envelope detection.

There has been a growth in literature applying superresolution techniques. In particular, there has been a focus towards high frequency acquisition, with SVD filtering, in vivo $[12,19,20]$. In this work high frequency is defined as transmit frequencies above the Sonovue ${ }^{\mathrm{TM}} \mathrm{MB}$ resonance (1- $3 \mathrm{MHz}$ ). Song et al., 2018, also recently showed the use of DI for the visualization of rabbit kidney vasculature at high acquisition frequency of $8 \mathrm{MHz}$ [21]. This paper aims to explore the parameter space, through simulation, in a way that would not be feasible in vivo. There is a need to understand how the extracted signals are affected by the combination of various detection algorithms and inter-dependent acquisition, processing and physiological parameters. Due to the challenges of creating a microvasculature phantom and the practical requirements of exploring such a vast parameter space, simulation has been used to provide a ground truth validation.

Within SVD filtering itself there are several choices to make. A main challenge is determining the singular value thresholds. These can be determined empirically [22]; or by choosing cutoffs based either on Doppler frequencies of the singular vectors or the singular value curve turning points [23]. Stack size has been shown to affect CTR of the filtered images [23-25]. There is no universally agreed stack size: much of the recent literature does not clearly state the size being processed. A better understanding of how SVD processing parameters might affect super-resolution is required. In this work, stack size and eigenvalue cut-off choices are considered.

However, before the processing is considered, decisions about the acquisition parameters must be made. In particular, the influence of frequency in super-resolution imaging is multifaceted. Increasing frequency will limit the depth penetration of the ultrasound beam through tissue. In conventional imaging, this makes it harder to reconstruct high resolution images at depth. Additionally, the behaviour of contrast agents is frequency dependent [26]. The low resonance frequency of MBs mean that super-resolution should not be fundamentally limited by depth. However, non-linear propagation of the beam through tissue and MBs can reduce the CTR [27]. This is especially problematic as the harmonic signal is already a fraction of the intensity of the linear MB signal. The bandwidth limitations of transducers are also an important consideration when comparing techniques. The influence of transmit frequency with and without including the effect of the transducer bandwidth in the model is explored in this work. Investigations without inclusion of the transducer bandpass allow a more general investigation of how properties such as frequency dependent attenuation and nonlinear propagation may affect the different methods. Including the bandpass filtering then highlights the importance of probe sensitivity for each method.

The influences of physiological factors are especially important to understand as they cannot be controlled. The visualization of tumor microvasculature is a particularly challenging environment to image and a potential application for super resolution [28, 29]. Blood flow in the tumor microvasculature can be an order of magnitude less than healthy vessels - with speeds less than $1 \mathrm{~mm} / \mathrm{s}$ for vessels up to $60 \mu \mathrm{m}$ in diameter [30]. Tumor microvasculature flow is changeable, even briefly stopping or reversing direction [31]. Structurally, tumor vessels are tortuous and form a complex 3D structure [32]. Nonetheless, accurate longitudinal and noninvasive imaging is essential for the development of therapeutic agents [33]. Thus this work investigates the dependence of each 
detection method due to $\mathrm{MB}$ speeds between $0 \mathrm{~mm} / \mathrm{s}$ and 2 $\mathrm{mm} / \mathrm{s}$, and studies the effect of flow direction bound between lateral flow (parallel to the transducer) and axial flow (perpendicular to the transducer).

This work introduces a simulation environment where nonlinear and linear detection methods for super resolution ultrasound can be quantitatively compared. Currently, superresolution ultrasound can and is performed over a huge parameter space. The factors affecting methods of extracting MB signals from the surrounding tissue have not been previously explored. The simulation has been designed with the intention of quantifying how factors such as MB size, frequency and blood velocity will affect how accurately and precisely the extracted MB signals can be localized using the different detection techniques. Although many of the results will be applicable more generally, physiological parameters relevant to the specific clinical example of visualization of tumor microvasculature have been chosen here. A series of simulations will investigate how the choice of detection technique may blur or distort the super-resolution images. The following methods section will first outline the overall simulation workflow and then detail the specifics of each simulation.

\section{METHODS}

\section{A. Use of $k$-Wave}

$\mathrm{k}$-Wave is a simulation platform which solves coupled nonlinear differential equations which describe the non-linear wave propagation through media [34]. $\mathrm{k}$-Wave models have been shown to closely replicate experiment [35]. The distinguishing feature of $\mathrm{k}$-Wave is how the differential terms are calculated. Instead of using a finite difference or finite element approach, which is computationally unfeasible for the required degree of accuracy, the global Fourier spectral method is used. This involves spatially discretizing the field of view by representing the space as a grid. Temporally the simulation is discretized by defining a time step associated with this grid. Mass and velocity sources can be defined on the grid and the resulting acoustic field can be sampled across the field of view by defining sensor positions. Further details of how the k-Wave package models wave propagation is provided in Treeby et al. (2012), and a simple diagram of the simulation geometry is provided in Figure 3(A). To our knowledge, k-Wave has not previously been combined with $\mathrm{MB}$ simulation. Outlined here are the parameters used in the subsequent simulations.

\section{- Discretization}

The grid spacing must be sufficiently fine to capture the highest frequencies of the signal components. In addition, discretization impacts the numerical error. Convergence tests were performed, and the error minimized by setting a fine spatial grid spacing of 6 points per wavelength for each center frequency.

Stability is ensured by an appropriate choice of time step. The Courant-Friedrichs-Lewy (CFL) number is introduced to ensure that the temporal and spatial discretization are appropriately related [36]. This dimensionless number is:

$$
\mathrm{CFL}=c_{\max } \frac{\Delta t}{\Delta x}
$$

where $c_{\max }$ is the maximum sound speed, $\Delta \mathrm{t}$ is the temporal discretization and $\Delta \mathrm{x}$ is the spatial discretization. The CFL number was set to be 0.05 following convergence testing to ensure that any numerical error was negligible.

- Tissue

Tissue was modelled based on the soft tissue acoustic properties provided by Azhari, 2010, see Table 1, [37].

Table 1: Bulk tissue properties from Azharia (2010).

\begin{tabular}{|c|c|}
\hline Tissue Parameters & Values \\
\hline Speed of Sound $(c)$ & $1570 \mathrm{~ms}^{-1}$ \\
Density $(p)$ & $1050 \mathrm{kgm}^{-3}$ \\
Power Law Prefactor $(\alpha)$ & $1 \mathrm{db}\left(\mathrm{MHzcm}^{-1}\right.$ \\
Power Law Prefactor $(y)$ & 1.05 \\
B/A Non-linear Exponent $(B / A)$ & 7.4 \\
\hline
\end{tabular}

Inhomogeneity to model boundaries between tissue and blood was introduced by assigning regions across the frame to equal the acoustic properties of blood as shown in Figure 3(C). Heterogeneity on the sub-resolution scale was introduced by defining a number of scatterer positions, see Figure 3(D). The acoustic properties of these scatterers were normally distributed with a mean of the bulk tissue values and a standard deviation of $0.8 \%$ of the mean. The number of scatterers per resolution cell was 10 for each center transmit frequency to ensure a fully developed speckle pattern [38].

\section{- Transducer Design}

Active elements of a linear transducer were modelled as time varying velocity sources. Grid points corresponding to transducer elements contained velocity sources and grid points corresponding to kerf regions were left empty as shown in Figure 3(A). Plane wave transmission of a single angle was modelled by setting an axial focus of infinity with all elements firing simultaneously. The apodization across the transducer was implemented by applying a Hamming filter across the transducer aperture.

Finally, the receive transducer was modelled by positioning sensors at the element centers. The received signals were bandpassed filtered using realistic transducer sensitivities detailed later. The frames were downsampled so that the time discretization matched the $50 \mathrm{MHz}$ sampling rate of the experimental ULA-OP system [39]

\section{B. Incorporation of Microbubble signal}

\section{- MB Parameters}

The MB response can be simulated using existing models which describe MB dynamics [40, 41]. All present MB models are derived from the Rayleigh-Plesset equation [40]. For this work the Marmottant model has been used [42] due to its ability to predict the highly non-linear response which can be experimentally observed [43]. This work focussed on modelling Sonovue MBs. Five different radii were modelled between $0.5 \mu \mathrm{m}$ and $5 \mu \mathrm{m}$ to sample the size distribution reported by Gorce et al. (2000) [44]. 


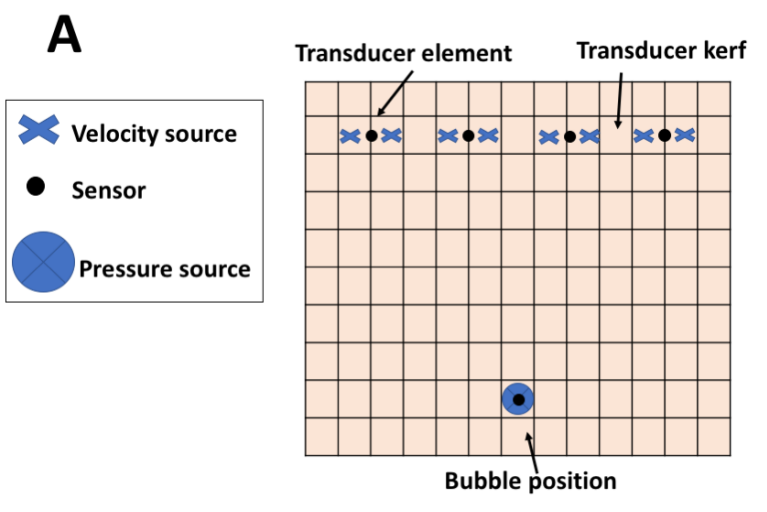

B

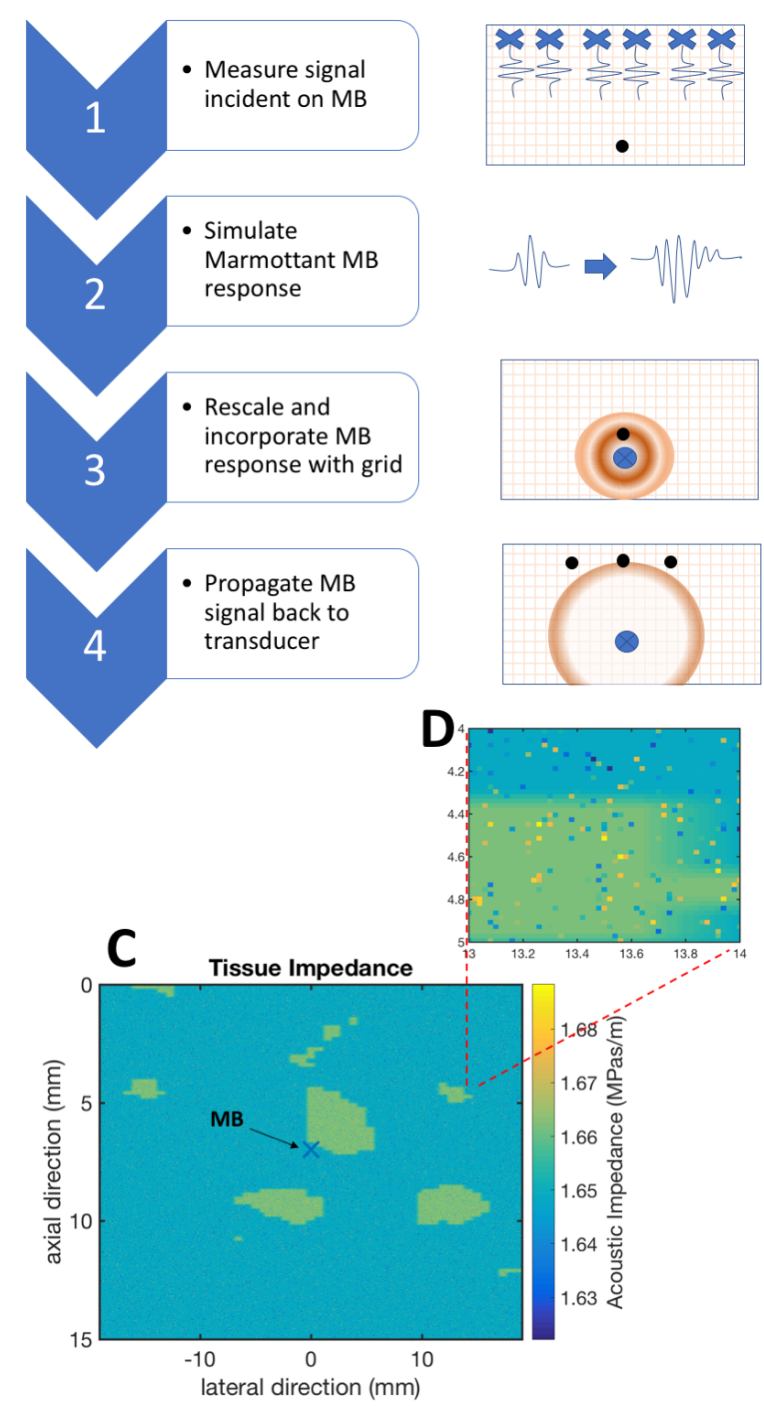

Fig. 3. MB simulation protocol. (A) Schematic of simulation geometry showing the sensors and sources on the grid. (B) Flow diagram of MB simulation steps. (1) A plane wave was generated by a line of mass sources. The resulting pressure was measured at the MB position. (2) The Marmottant response was determined. (3) This response was rescaled for incorporation with the grid. (4) The MB signal was propagated back to the sensor positions. (C) Impedance values of tissue, showing MB position and bulk inhomogeneity. (D) Inset shows the scatterer distribution generating speckle.
Table 2: MB properties from literature used in simulation - [45] van der Meer, Dollet et al. 2007; [46] Tu, Guan et al. 2009; [37] Azhari, H. (2010); [47] International Civil Aviation Organization (1993).

\begin{tabular}{|c|c|c|}
\hline Model Parameter & Value & Reference \\
\hline Bubble surface tension $(\sigma)$ & $\sigma\left(R_{0}\right)=0.072 \mathrm{Nm}^{-1}$ & {$[45]$} \\
Gas exponent $(\kappa)$ & 1.07 & {$[45]$} \\
Dynamic viscosity $(\mu)$ & $2 \times 10^{-3} \mathrm{Nsm}^{-2}$ & {$[45]$} \\
Shell elasticity $(\chi)$ & $0.3 \mathrm{Nm}^{-2}$ & {$[46]$} \\
Shell viscosity $\left(\kappa_{\mathrm{S}}\right)$ & $3.2 \times 10^{-9} \mathrm{kgs}^{-1}$ & {$[46]$} \\
Blood Speed of Sound $(c)$ & $1575 \mathrm{~ms}^{-1}$ & {$[37]$} \\
Blood Density $\left(p_{1}\right)$ & $1055 \mathrm{~km}^{-3}$ & {$[37]$} \\
Ambient Pressure $\left(P_{0}\right)$ & $101325 \mathrm{kgm}^{-3}$ & {$[47]$} \\
\hline
\end{tabular}

The MB response has been modelled using the Marmottant model [42]:

$$
\begin{gathered}
p_{l}\left(R \ddot{R}+\frac{3}{2} \dot{R}^{2}\right)=\left(P_{0}+\frac{2 \sigma\left(R_{0}\right)}{R_{0}}\right)\left(\frac{R}{R_{0}}\right)^{-3 \kappa}\left(1-\frac{3 \kappa}{c} \dot{R}\right)- \\
P_{0}-\frac{2 \sigma(R)}{R}-\frac{4 \mu \dot{R}}{R}-\frac{4 \kappa_{s}}{R^{2}}-P(t) .
\end{gathered}
$$

where $P(t)$ is the pressure response the MB experiences, $R$ is the $\mathrm{MB}$ radius, and the equilibrium radius is given by $R_{0}$ (all other parameters are defined in Table $2[37,45-47])$. This involves a radii dependent surface tension term:

$$
\sigma(R)=\left\{\begin{array}{c}
0 \text { if } R \leq R_{\text {buckling }} \\
\chi\left(\frac{R^{2}}{\left.R_{\text {buckling }}^{2}-1\right)} \text { if } R_{\text {buckling }} \leq R<R_{\text {ruptured }}\right. \\
\sigma_{\text {water }} \text { if } R \geq R_{\text {ruptured }}
\end{array}\right.
$$

where the limits were:

$$
\begin{gathered}
R_{\text {ruptured }}=R_{\text {buckling }}\left(1+\frac{\sigma_{\text {water }}}{\chi}\right)^{\frac{1}{2}} \\
R_{\text {buckling }}=0.99 \cdot R_{0 .}
\end{gathered}
$$

Solving Equation 2 generated the transient radial response $(R)$, shell velocity $(\dot{R})$ and shell acceleration $(\ddot{R})$. Thus, the pressure emitted from the scatterer could be determined from [48]:

$$
P=\frac{p_{l}}{R_{p}}\left(R^{2} \ddot{R}+2 R \dot{R}^{2}\right)-\left(\frac{R}{R_{p}}\right)^{4}\left(\frac{p_{l} \dot{R}^{2}}{2}\right)
$$

where $R_{p}$ corresponds to the distance from the bubble.

Figure $3 \mathrm{~B}$ outlines the four steps used to incorporate the Marmottant model and k-Wave simulation.

1. Over the parameter space the propagation of a transmit pulse 3 cycles in length and with a mechanical index (MI) of 0.1 was modelled. Prior to the transmit wave reaching the bubble, the pulse will have experienced frequency dependent attenuation and non-linear propagation through the tissue. This was included by simulating the transmit pulse and recording the waveform at each MB position using a k-Wave sensor.

2. The MB response was modelled by using the signal 
received from step 1 as the input to the Marmottant model.

3. The Marmottant pressure response was then added as a mass source to the mass conservation equation at the MB position. This generated a monopole field as if from a radially oscillating volume [49]. The MB size is not negligible compared to the grid dimensions. This meant the MB pressure response could not be accurately represented by a mass source at a single point on the grid. Rescaling of the pulse was required so that the amplitude matched that expected from Equation 6 at a given distance from the MB position. This was done by determining $P$ from Equation 6 using $R p=50 \mu \mathrm{m}$. This was then multiplied by a constant factor to approximate the pressure amplitude at $R p=\mathrm{R}_{0}$. This approximate MB response was added as a point source in the grid and propagated a distance of $50 \mu \mathrm{m}$ from the MB position. The pulse was then compared to the theoretical amplitude given by Equation 6 when $R p$ $=50 \mu \mathrm{m}$. The input was rescaled to ensure the simulated value at $50 \mu \mathrm{m}$ matched the theoretical value. This specific value of $R p$ was chosen arbitrarily as a value which was an order of magnitude greater than any variations between MB sizes, whilst still being small enough to enable kWave simulations to be performed in a reasonable time.

4. The nonlinear propagation of the rescaled MB response to the receive transducer was then modelled using k-Wave. The receive transducer was modelled using sensors at the element centers.

\section{- MB Movement}

Scatterer and source positions can only be defined at grid points in k-Wave. Sensors can be defined at any position in the simulation plane. Since bubble movements were generally less than $1 \mu \mathrm{m}$ between frames, and grid dimensions on the order of tens of microns, movement was introduced by shifting the sensor positions and source phase rather than scatterer or source positions. All data was acquired at a pulse repetition frequency (PRF) of $1000 \mathrm{~Hz}$. The MB and tissue response were simulated separately and then coherently combined in order to simulate relative motion on the micron scale.

\section{- Noise}

This was achieved by generating white Gaussian noise using a built in MATLAB function $(\operatorname{awgn}())$. The signal to noise ratio (SNR) for the simulation was chosen to approximate that calculated from experimental data. The reference experiment used a suspension of $0.3 \mathrm{ml}$ of Sonovue ${ }^{\mathrm{TM}}$ (Bracco) MBs diluted using $50 \mathrm{ml}$ of ultrapure water (Milli-Q ${ }^{\circledR}$ ) and drawn through a $200 \mu \mathrm{m}$ tube at a flow rate of $15 \mu \mathrm{l} / \mathrm{min}$. The flow phantom was imaged at a transmit center frequency of 4 MHz using the LA332 probe (Esaote) and ULA- OP (Univ. degli Studi di Firenze, Florence, Italy). Single plane waves of phase +1 were acquired and regions of interest corresponding to tube and MB (signal) and noise defined on the radiofrequency $(\mathrm{RF})$ data. SNR was defined by:

$$
S N R=10 \log _{10}\left(\frac{\left\langle A_{\text {signal }}^{2}\right\rangle}{\left\langle A_{\text {noise }}^{2}\right\rangle}\right)(7)
$$

where $A_{\text {signal }}$ and $A_{\text {noise }}$ are the signal and noise amplitude respectively. The SNR for this experiment was calculated to be $26.3 \mathrm{~dB}$. In order to compare the different MB sizes and parameter sets the noise level was kept constant (the SNR was allowed to vary). The amplitude of the noise was determined by fixing the SNR of the modelled RF image of a 2 $\mu \mathrm{m} \mathrm{MB}$ in tissue at $4 \mathrm{MHz}$ to be $26.3 \mathrm{~dB}$.

\section{Post Processing}

\section{- Beamforming}

The beamforming involved finding the signal at each pixel, due to all scatterers, based on the relative delays of the received signals across the transducer assuming a homogeneous sound speed [50]. A Hamming weighting across the transducer elements was also incorporated.
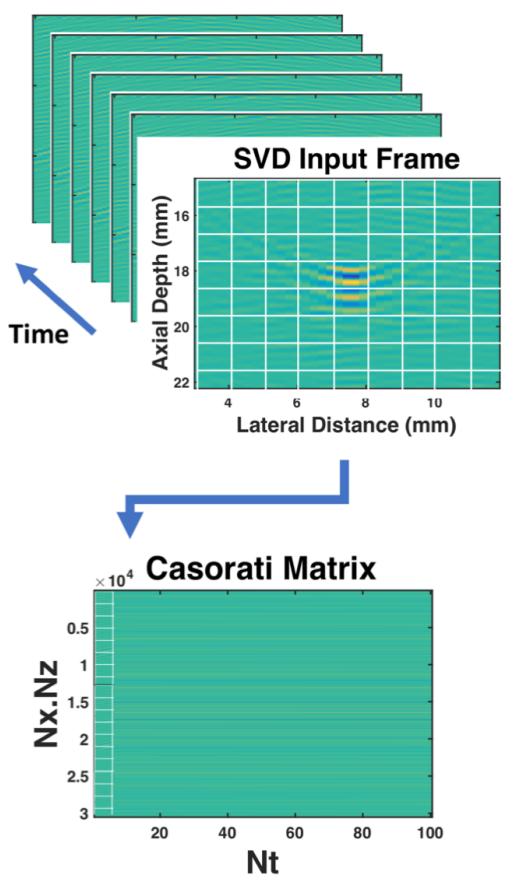

Fig. 4: Generation of Casorati matrix for SVD. Each frame of spatial information is reformed as a column vector of the Casorati matrix.

\section{- $\quad$ MB Detection methods}

Singular Value Decomposition Filtering.

Demené et al., (2015) describes the method of SVD for contrast enhanced ultrasound in detail [22]. The technique is outlined briefly here. First, the spatial and temporal information is combined by sorting the data into a Casorati matrix, (S). This is where each frame is vectorized and added as a column as shown in Figure 4. This work uses beamformed RF data (before Hilbert transform). SVD factorizes this Casorati matrix to:

$$
S=U D V^{*}
$$

where columns of $U$ are spatial singular vectors and corresponding columns of $V$ are the associated temporal vectors. $U$ and $V$ are orthonormal. $D$ is a diagonal matrix, where 
the elements are the singular values which decrease as the column number increases. They illustrate the relative contribution to the overall signal of each singular vector pair.

A spatial singular vector re-ordered back to $N z$ rows x $N x$ columns can be thought of as a virtual image extracted from the data, $U_{i}(x, z)^{\text {virtual }}$. The corresponding temporal singular vector controls how each of the pixel amplitudes vary together over time. For MB detection a lower singular value threshold is set $(l)$, below which their spatial and temporal signal contributions are discarded as unwanted tissue signal and an upper threshold is set $(u)$ beyond which the information is discarded as unwanted noise. The filtered signal is given by:

$$
\operatorname{Signal}(x, z, t)_{\text {filtered }}=\sum_{i=l}^{u} U_{i}(x, z)^{\text {virtual }} D_{i} V_{i}(t) \text {. }
$$

Computationally this was performed using the MATLAB economy-size SVD function $(\operatorname{svd}())$.

\section{Pulse Inversion}

Pulse inversion involved the summation of temporally adjacent positive and negative frames of simulated RF data before beamforming and envelope detection. This extracted any non-linear signal and changes due to movement [18].

\section{Differential Imaging}

Differential images were produced by the subtraction of temporally adjacent positive frames as in Desailly, Couture et al. (2013). Simulated RF frames were processed before beamforming and envelope detection. Signal was generated by movement between frames.

\section{Quantification}

\section{- Contrast to Tissue Ratio}

Figure 5(A-B) show the regions of interest used to determine the CTR. The regions of interest for $\mathrm{MB}$ and tissue signal have different axial positions due to reduced lateral resolution causing MB lobes to mask tissue breakthrough for some parameter sets. Thus, it was simpler to define the regions as shown in Figure 5(A-B) and compare maximum pressure values for the tissue $\mathrm{V}_{\text {tissue }}$ and $\mathrm{MB}$ signal $\mathrm{V}_{\text {bubble [51]: }}$

$$
C T R=20 \log _{10}\left(\frac{V_{\text {bubble }}}{V_{\text {noise }}}\right)
$$

The noise and localization process were repeated 20 times. 20 repetitions were empirically chosen as this generated a small spread of CTR values about the mean for the chosen noise level. The accuracy was determined as the mean of the bias between the localized and known MB positions and the precision by the standard deviation. This quantification was performed for the first output frame of each detection method so that the MB position in the field of view (FOV) was constant. It was also empirically observed that SVD filtering can cause the image intensity to fluctuate over the stack. This fluctuation causes the signal to drop from a maximum at $\mathrm{t}=0$ to a minimum corresponding to the center of the stack size, and then back to a maximum at the final frame of the stack. As this temporal fluctuation will depend on how exactly SVD is implemented, for example a moving temporal window may be affected less than the more typical application to a single stack, the first frame was chosen to compare PI and DI with the best case CTR for SVD.
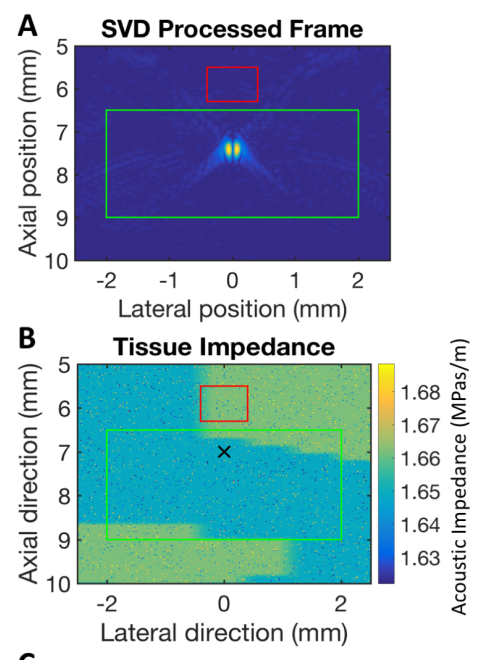

C

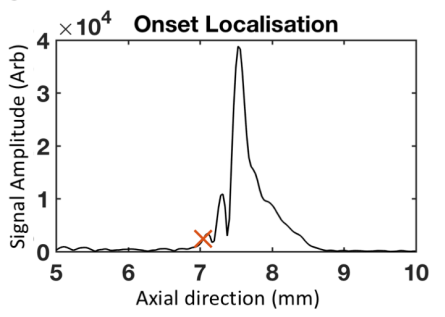

Fig. 5: Signal quantification in terms of CTR and onset localization. (A) These are shown on an example SVD processed frame, with the tissue region of interest and $\mathrm{MB}$ region of interest represented by the red box and green box respectively. (B) The ROIs are shown on the map of the tissue impedance, with the MB position shown by a cross. These regions were chosen to ensure $\mathrm{MB}$ lobe signal was not included across the parameter range. (C) Axial profile of MB signal, after finding the lateral position using centroiding, where the cross shows the axial onset localization.

\section{- Localization}

The extracted signals were envelope detected using a Hilbert transform. Localization method choices were tested in Christensen-Jeffries et al., (2017), and the onset method introduced [52]. This was shown to have the best axial localization accuracy in both simulation and experiment. The onset method involves first finding the centroid of the beamformed signal. The lateral centroid component is used as the lateral localization. The centroid is a reasonable indicator of the lateral position of the MB because the PSF is not asymmetrically affected by variation between MB sizes, asymmetry of the time, and duration in the lateral direction [52]. The two axial RF lines about this centroid position are averaged and the axial position found by choosing the start of the pulse at the point three standard deviations above the noise mean on this averaged signal. The axial profile is shown in Figure 5(C). For this work, a threshold of 4 standard deviations was chosen. This was because the onset method was developed at a specific set of parameters, using a higher threshold here enabled fairer comparison across these three detection techniques and wider parameter space. 


\section{E. Specific Simulations}

Simulation Characterization:

- MB frequency response:

The pressure response of MBs modelled using the Marmottant model and chosen MB parameters was determined over the frequency range 1-15 MHz. This enabled easier interpretation of later results, namely to highlight any differences introduced by the processing techniques. For each frequency a Gaussian pulse 3 cycles in length and mechanical index (MI) of 0.1 was used as the input to the Marmottant model.

- SVD stack size and filter order:

To determine a stack size which generates the best CTR for the SVD experiments, 1000 frames for a MB moving laterally at $0.5 \mathrm{~mm} / \mathrm{s}$ were simulated using a center transmit frequency of $6 \mathrm{MHz}$. These were filtered using varying stack sizes and eigenvalue cut-offs.

\section{Frequency dependence:}

- Transmit Center Frequency

Several different commercially available transducers will be able to image at the same transmit center frequency. However, differences in geometry and sensitivity of these transducers will mean that the resulting images are different for different probes. To achieve the most general comparison of the detection methods over different transmit frequencies a linear array was designed for each center frequency $(1.5 \mathrm{MHz}, 2 \mathrm{MHz}, 3 \mathrm{MHz}$, $4 \mathrm{MHz}, 5 \mathrm{MHz}, 6 \mathrm{MHz}$ and $7 \mathrm{MHz}$ ). The ratio of pitch size to transmit wavelength was set to a constant value of 0.61 to avoid the generation of side lobes. The aperture size was also set to constant value due to its impact on lateral resolution. Keeping the pitch to kerf ratio constant meant that the number of elements of each transducer ranges from 16 at $1 \mathrm{MHz}$ to 112 at $7 \mathrm{MHz}$. These values were chosen to correspond to experimental work using 64 elements of the LA332 probe (Esaote, Italy) with the ULA-OP (Universit degli Studi di Firenze, Italy) system at $4 \mathrm{MHz}$.

The influence of frequency was then determined for each processing method for a MB flowing laterally at $0.5 \mathrm{~mm} / \mathrm{s}$.

- Influence of Transducer Sensitivity

To simulate the more practical situation of using commercially available transducers of a particular geometry and frequency sensitivity the following simulations were performed using the properties of the PA230 (1-4 MHz) and LA332 (1-4 MHz) probes. Reported pitches for PA230 and LA332 are $170 \mu \mathrm{m}$ and $245 \mu \mathrm{m}$. Due to the requirement of defining these on the grid, the values provided in Figure 6 were used.

This enabled investigation of how transducer sensitivity, and position of transmit center frequency within the probe bandpass, have different implications for different detection methods.

Now the frequency could be fixed to one determined appropriate for each technique.
Tumor Physiological Environment

- Dependence on speed:

The dependence of CTR and localization accuracy and precision were determined for a range of speeds $<2 \mathrm{~mm} / \mathrm{s}$ for lateral motion using a probe and frequency suitable for each technique.

- Dependence on direction:

The dependence of CTR and localization accuracy and precision were determined for a range of flow directions at a speed of $0.5 \mathrm{~mm} / \mathrm{s}$, again using a probe and frequency suitable for each technique.
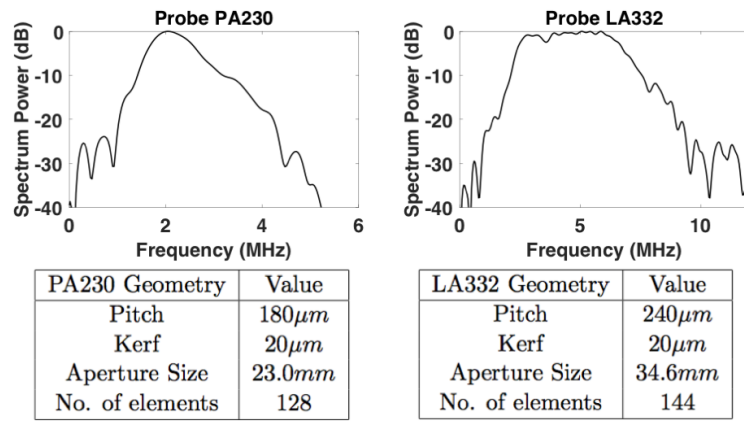

Fig 6: Simulated transducer properties. Parameters of simulated transducers incorporated on the grid based on the PA230 and LA332 probes. 


\section{RESULTS}

\section{A. Simulation Characterisation}

- Marmottant Model:

Figure 7(A) shows the predicted behaviour of each MB size over the frequency range 1-15 MHz, where the MI was kept constant over the frequency range. Figure 7(B) shows the expected size distribution of Sonovue from Gorce et al. (2000) which is used to find a weighted average of quantitative measures. As expected, the scattering generally increases in amplitude as frequency increases due to the increased scattering cross-section. MB resonance behaviour can be observed at low frequencies using these pulse parameters.
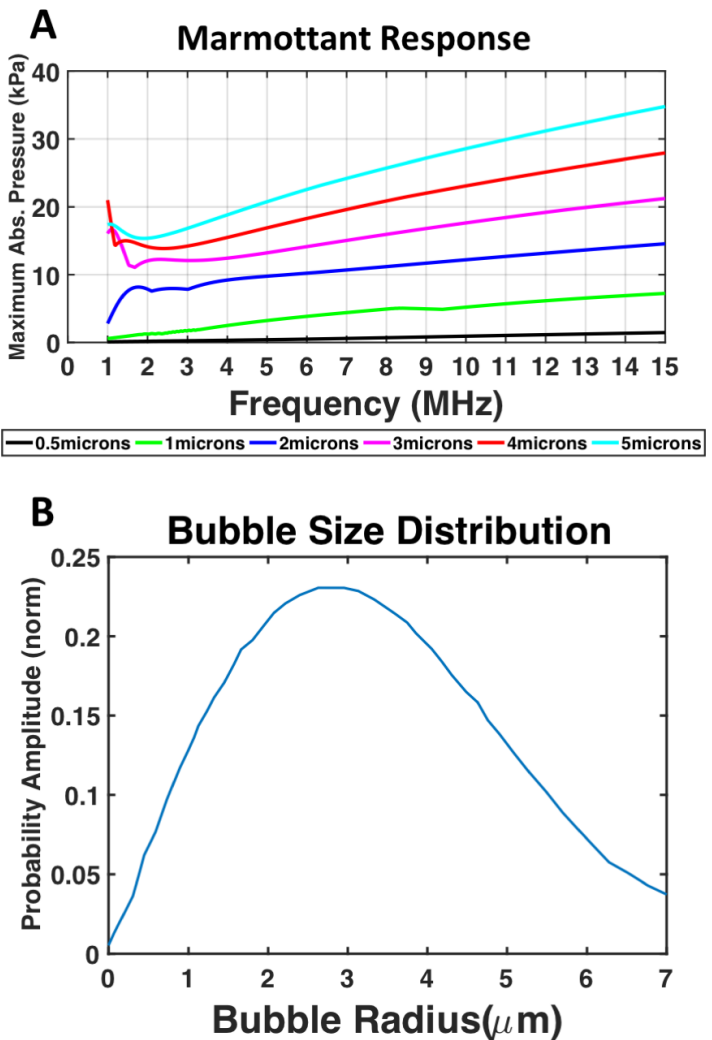

Fig. 7: Microbubble frequency dependence and size distribution. (A) shows the peak absolute pressure from simulated MBs recorded across the center transmit frequency range $1-15 \mathrm{MHz}$ for $5 \mathrm{MB}$ radii and constant MI of 0.1. (B) shows the expected size distribution of Sonovue extracted from Gorce et al. (2000) (B).

- $\quad$ SVD Stack Size:

Figure 8 investigates the influence of stack size on a $2 \mu \mathrm{m}$ radius $\mathrm{MB}$ moving at $0.5 \mathrm{~mm} / \mathrm{s}$ laterally using a pulse with center transmit frequency of $6 \mathrm{MHz}$. The CTR rises until a stack size of 250 frames is reached. For this set of parameters, the CTR began to fall at stack sizes beyond 250 frames. Figure 8(B) shows that a larger stack size allows the signal to spread over a wider range of eigenvalues. Figure $8(\mathrm{C})$ shows that the CTR cannot be improved for adjusting the lower singular value cut-off for each stack size.

To achieve the best CTR for SVD with the lowest acquisition time, the following SVD demonstrations were performed using 250 frames and removing the signal associated with the first singular value and those values $>14$ which were associated with noise.
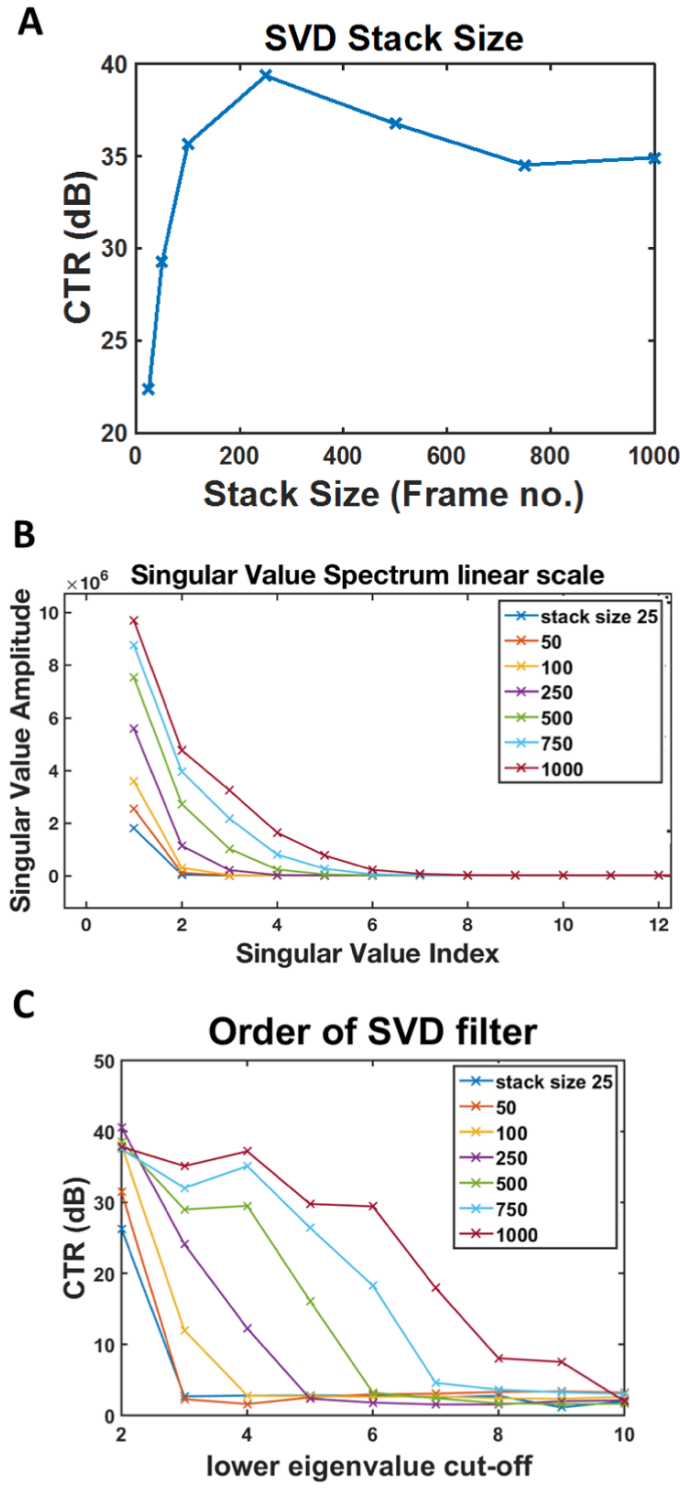

Fig. 8: Effect of SVD stack size. (A) Effect of stack size on CTR when filtering a $2 \mu \mathrm{m}$ MB moving laterally at $0.5 \mathrm{~mm} / \mathrm{s}$. This shows that large stack sizes are not always beneficial. (B) Singular value spectrum at each stack size. As stack size increases the weightings of each component become more evenly shared. (C) CTR for each stack size as lower SVD eigenvalue cut-off is adjusted.

\section{B. Effect of Frequency}

Figures 9-11 show the effect of center transmit frequency and bandpass filtering on super-resolution in terms of CTR and localization precision.

Figure 9 shows the results without transmit or receive bandpass filtering to enable generalization of the results to different probes. This was to ensure that any trends due to varying center transmit frequency alone were not obscured by the introduction of filtering. Figure 9 (A) shows the trends of individual bubbles due to the growing interest of the influence of MB size on super-resolution [20]. These results are then presented as weighted averages in Figure 10(B), to show any 

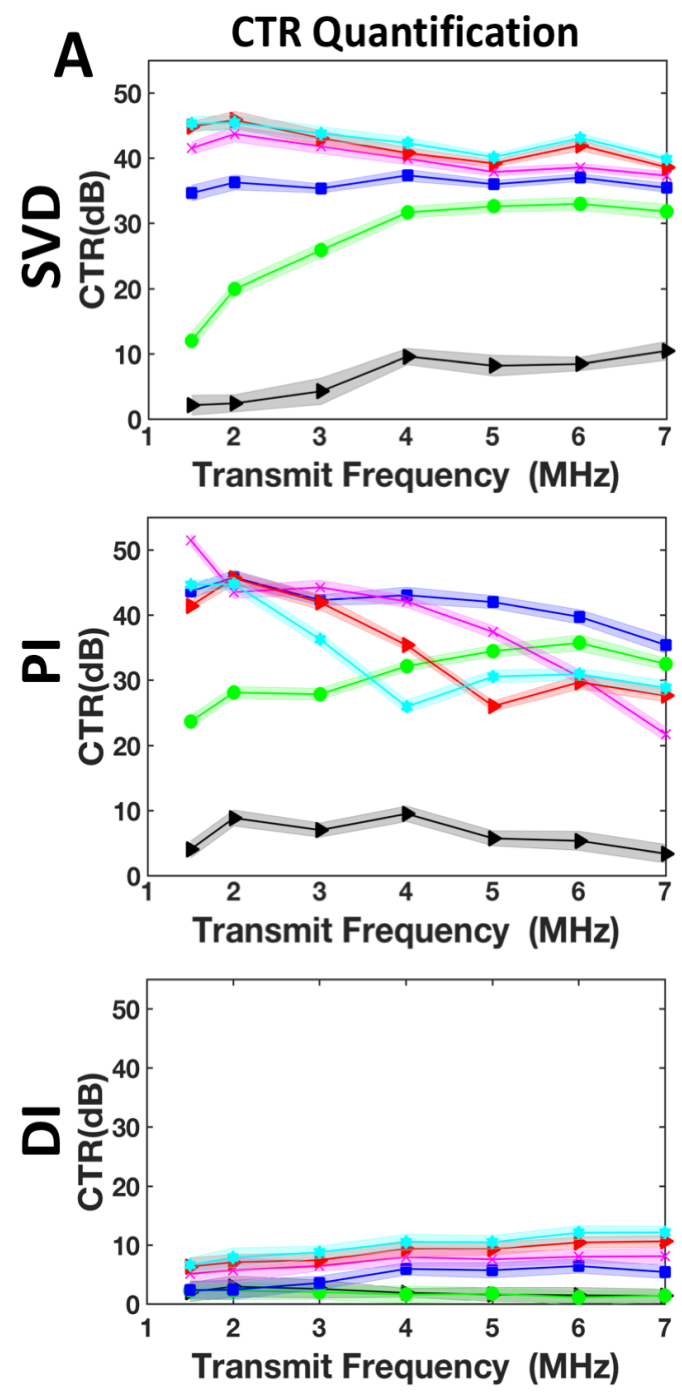

$0.5 \mu \mathrm{m} \bigcirc 1 \mu \mathrm{m} \square 2 \mu \mathrm{m} \times 3 \mu \mathrm{m}>4 \mu \mathrm{m}+5 \mu \mathrm{m}$

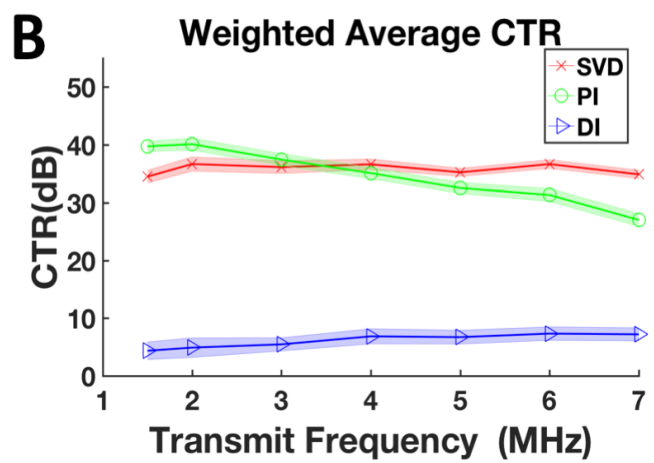

Fig. 9: CTR dependence on center transmit frequency. (A) shows the CTR dependence of each MB radii on acquisition center frequency for MBs flowing laterally at $0.5 \mathrm{~mm} / \mathrm{s}$ for SVD (top), PI (middle) and DI (bottom). (B) presents the CTR weighted average over the MB distribution for all three detection methods.

overall frequency dependence of the detection techniques. The weighted average was calculated by scaling the result for each $\mathrm{MB}$ size according to the expected Sonovue ${ }^{\mathrm{TM}}$ size distribution as reported in Gorce et al. (2000) before summing together.

Figure 10 shows how the localization precision depends on frequency due to the changing CTR and size of initial point spread function.

Finally, Figure 11 introduces bandpass filtering that matches the sensitivity from commercially available probes. This is to explore whether some techniques are more affected than others by the probe sensitivity and position of center frequency in the bandwidth.

\section{- Low Frequency Acquisition:}

Figure 9(A and B) shows that PI has the greatest CTR at the lowest frequencies. Individual bubbles contribute differently to the total CTR for each processing technique. CTR increases with increasing radii for SVD and DI, see Figure 9(A). Due to resonance behaviour this trend is not true for PI, also shown in Figure 9(A). Figure 9(B) shows that the weighted average CTR of PI decreases as the MBs exhibit less non-linear behaviour at higher frequencies. PI generated a CTR improvement of $3.4 \mathrm{~dB}$ at a transmit frequency of $2 \mathrm{MHz}$ over SVD and $36.2 \mathrm{~dB}$ over DI.

Figure 10 shows the high axial and lateral precision of PI at low frequencies. The average axial and lateral precision at 2 $\mathrm{MHz}$, for example, is $7.8 \mu \mathrm{m}$ and $1.7 \mu \mathrm{m}$ respectively for PI imaging.

Missing points on Figure 10 correspond to simulations where the MB signal was not sufficiently greater than the tissue/noise for the onset method to detect a MB. The results show that DI is not appropriate at frequencies $\leq 4 \mathrm{MHz}$ for the velocities used in this simulation.

Figure 11 shows the incorporation of a bandpass filter over the frequency range $1.5-4 \mathrm{MHz}$ for PI. This technique suffers
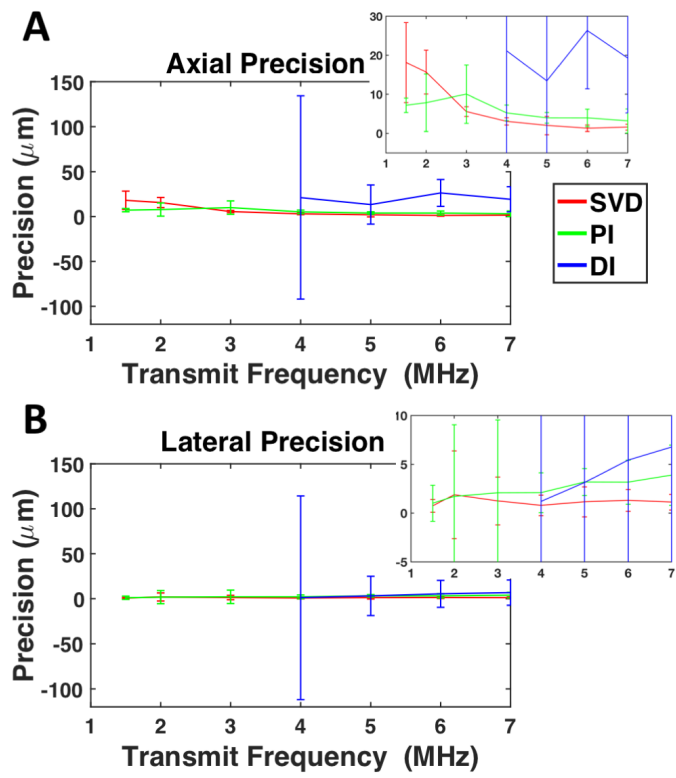

Fig. 10: Dependence of localization precision on center transmit frequency. (A) shows the average axial localization precision at each transmit center frequency for each of the MB detection methods. This is the standard deviation of the axial position measured over repeated simulations. The error bars represent the standard deviation of the weighted average, i.e. variation due to contributions from different $M B$ radii. (B) shows the average lateral localization precision for each MB detection method, where the inset is to visualize data points that have error bars that are much smaller than for DI. 
as the second harmonic frequency rises above the transducer sensitivity range. For most commercially available low frequency transducers, ensuring that transmit frequencies are at the lower end of the transducer sensitivity, to ensure sufficient sensitivity at the $2^{\text {nd }}$ harmonic, is crucial for PI.

- High Frequency Acquisition:

Figure 9(A) shows an upwards trend in CTR for SVD with frequency for the MB sizes $<2 \mu \mathrm{m}$ in radius. For larger MB radii, the CTR of the SVD processing is fairly constant over the frequency range. And Figure 9(B) shows that, overall, SVD does not significantly vary with frequency. As shown in Figure 9(B), DI showed improved CTR with increasing frequency with an increase of $2.98 \mathrm{~dB}$ at $6 \mathrm{MHz}$ compared to $1.5 \mathrm{MHz}$. However, it is clear that for these flow rates, SVD is preferable to DI across the frequency range.

Figure 10 shows that DI localization was only possible for frequencies $\geq 4 \mathrm{MHz}$. The localization precision plots for individual bubble sizes have not been presented here. Instead, Figure 10 shows the weighted average. Some MB radii did not generate sufficient CTR to enable localization, so did not contribute to the weighted average presented in Figure 10. Only MBs $\geq 3 \mu \mathrm{m}$ in radii generated sufficient CTR to enable localization for DI at center transmit frequencies $\geq 4 \mathrm{MHz}$. SVD and PI could localize all but the smallest MBs, $0.5 \mu \mathrm{m}$ radii, over the frequency range studied here.

\section{Bandpass Filter}
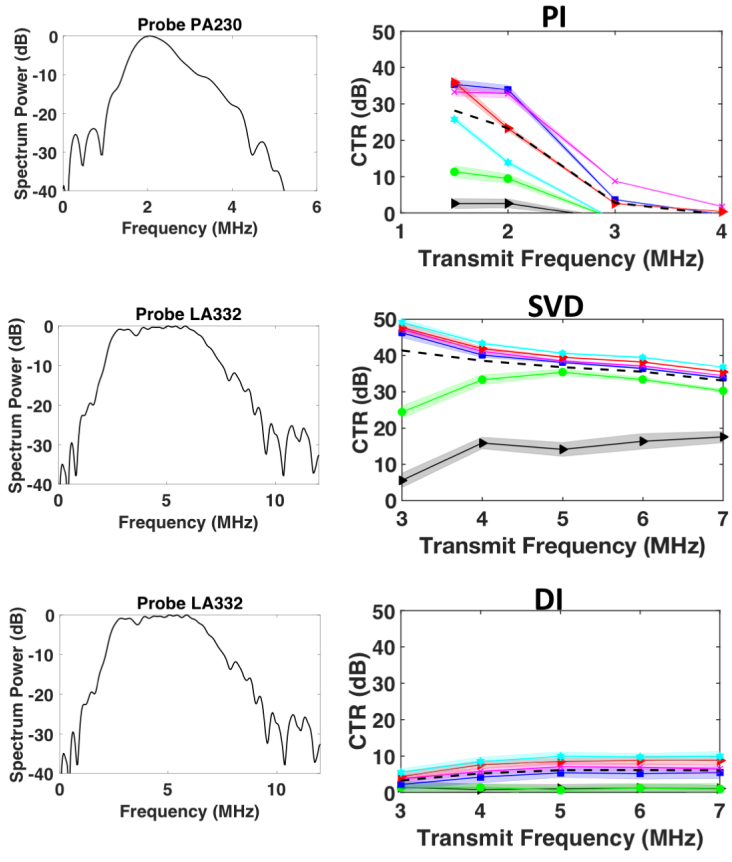

$>0.5 \mu \mathrm{m} \bigcirc 1 \mu \mathrm{m} \square 2 \mu \mathrm{m} \times 3 \mu \mathrm{m}>4 \mu \mathrm{m}+5 \mu \mathrm{m}-$ weighted average

Fig. 11: Effect of transducer bandpass filter. (Left) The bandpass of the modelled transducer based on PA230 (top) and LA332 (middle and bottom). (Right) Frequency dependence on CTR for PI (top), SVD (middle) and DI (bottom) when probe sensitivities are introduced.

Figure 11 shows that introduction of the bandpass filter has less effect on CTR for the linear techniques over the frequency range compared to PI.

For SVD, although average CTR is fairly constant over the frequency range, the improved axial and lateral precisions at higher frequencies motivates the use of SVD for high frequency applications, see Figure 10. The axial precision improves from $18.1 \mu \mathrm{m}$ at $1.5 \mathrm{MHz}$ to $1.6 \mu \mathrm{m}$ at $7 \mathrm{MHz}$.

\section{Velocity dependence}

For the following simulations, PI was performed at a frequency of $2 \mathrm{MHz}$ using the bandpass filter associated with the PA230 probe. DI and SVD were performed at a higher frequency of $6 \mathrm{MHz}$ using the bandpass filter associated with the LA332 probe. These center frequencies and probes were chosen for each technique considering the best average CTR and precision values determined in the previous section.
A
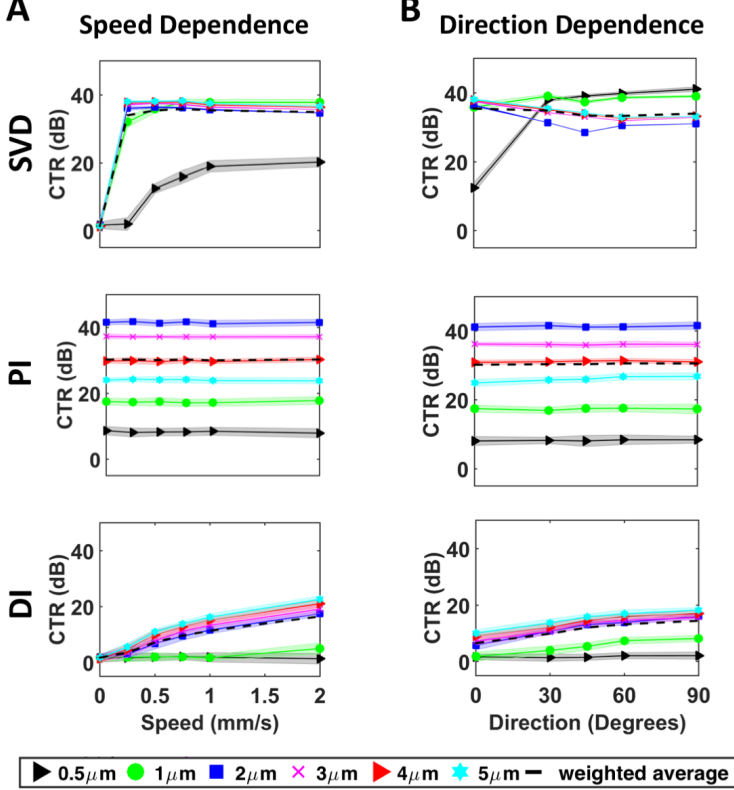

C

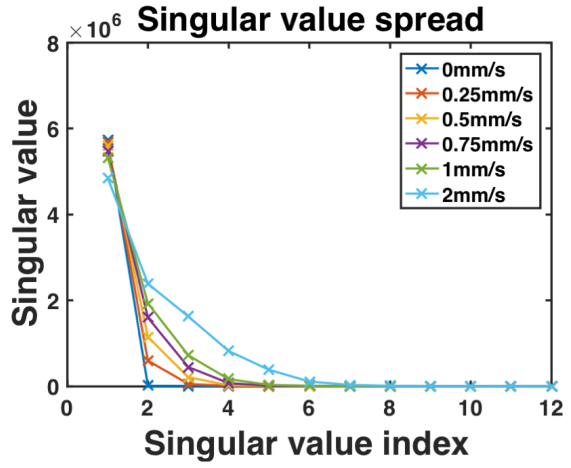

Fig. 12: CTR dependence on velocity (A) shows the CTR dependence of each MB radii and the weighted average on flow speed for MBs flowing laterally. (B) shows the CTR dependence on flow direction at a speed of $0.5 \mathrm{~mm} / \mathrm{s}$. (C) Spreading of data across singular vectors for varying lateral speed.

Figure 12 shows how CTR varies with MB flow speed and direction. Figure 13(A and C) are graphical visualizations of how the accuracy and precision values determined from the simulation results will affect SR images for different $\mathrm{MB}$ speeds and directions respectively. For each processing technique an example ground truth vessel is shown, where each $30 \times 30 \mu \mathrm{m}$ segment corresponds to a different velocity. Using the values for the weighted average of localization and 


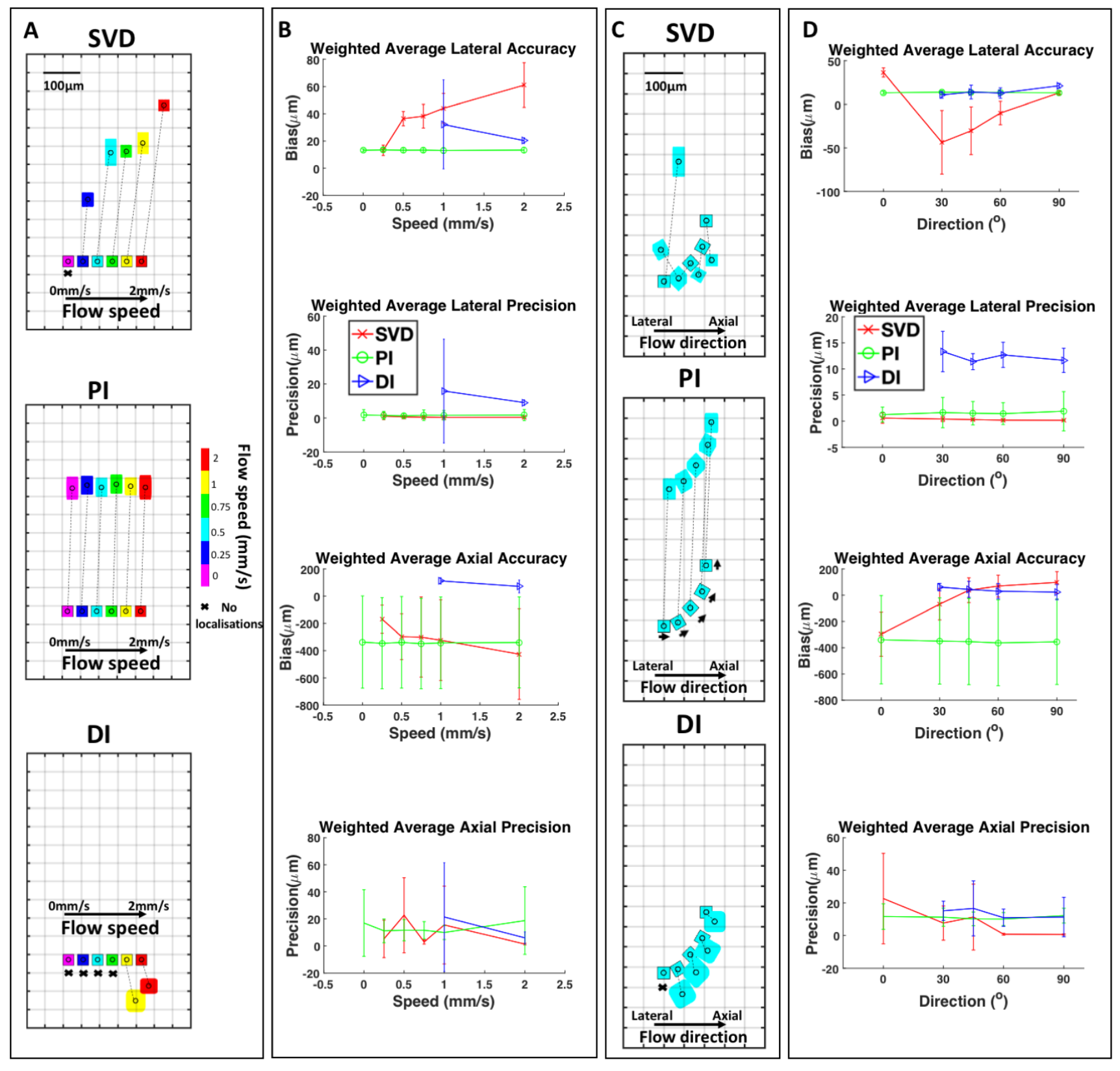

Fig. 13: Localization dependence on velocity. (A) Visualization of localization results over speed range $0-2 \mathrm{~mm} / \mathrm{s}$ for SVD (top), PI (middle) and DI (bottom). Scale is represented by a background grid $(50 \times 50 \mu \mathrm{m})$. The same ground truth vessel is presented at a depth of $7 \mathrm{~mm}$, with each vessel segment being represented by a square of size $(30 \times 30 \mu \mathrm{m})$. Each segment corresponds to a different MB flow speed. When CTR values are sufficient to generate localizations, the SR image is modelled by shifting each vessel segment by the localization accuracy determined from the simulation. If MB signal does not exceed the noise threshold a cross is shown to represent missing data. Localization precisions in lateral and axial directions are represented by increasing the width and height of the rectangle by the lateral and axial precision respectively, i.e. so that larger rectangles represent poorer precision. The center points of the corresponding ground truth and simulated segments are joined to highlight the translation. (B) Shows the accuracy and precision values determined from the simulation graphically. (C) Visualization of localization results over direction range $\left(0-90^{\circ}\right)$. The same ground truth vessel (of segment dimensions $30 \times 30 \mu \mathrm{m}$ ) is presented at a maximum depth of $7 \mathrm{~mm}$. Each segment corresponds to a different MB flow direction at a MB flow speed of $0.5 \mathrm{~mm} / \mathrm{s}$. When CTR values are sufficient to generate localizations the SR image is modelled by shifting each vessel segment by the localization accuracy determined from the simulation. The vertical extent of the rotated rectangle is increased by the axial precision and horizontal extent increased by the lateral localization precision. The center points of the corresponding ground truth and simulated segments are joined to highlight the translation. (D) Shows the accuracy and precision values determined from the simulation.

precision the simulated SR image is overlaid on the ground truth image. The simulated SR segments are shifted with respect to the ground truth by their axial and lateral bias. The height and width of the simulated SR image of these ground truths are increased by the axial and lateral localization precision respectively, with larger rectangles representing poorer precision. These same results for localization precision and accuracy are presented graphically in Figures 13(B and D), where the error bars represent the standard deviation from the weighted average over the different bubble radii.

- Applications with slowest/stationary MBs, varying flow rates or flow in different direction

Figures 12 and 13 both show that PI is less dependent on MB velocity compared to DI and SVD.

Figure 12 shows that CTR is constant over the $0-2 \mathrm{~mm} / \mathrm{s} \mathrm{MB}$ speed range and all flow directions for PI. In comparison, SVD and DI are not appropriate for stationary MBs. At $0 \mathrm{~mm} / \mathrm{s}$ the CTR drops to $0 \mathrm{~dB}$ for SVD and DI. This is also shown by the 

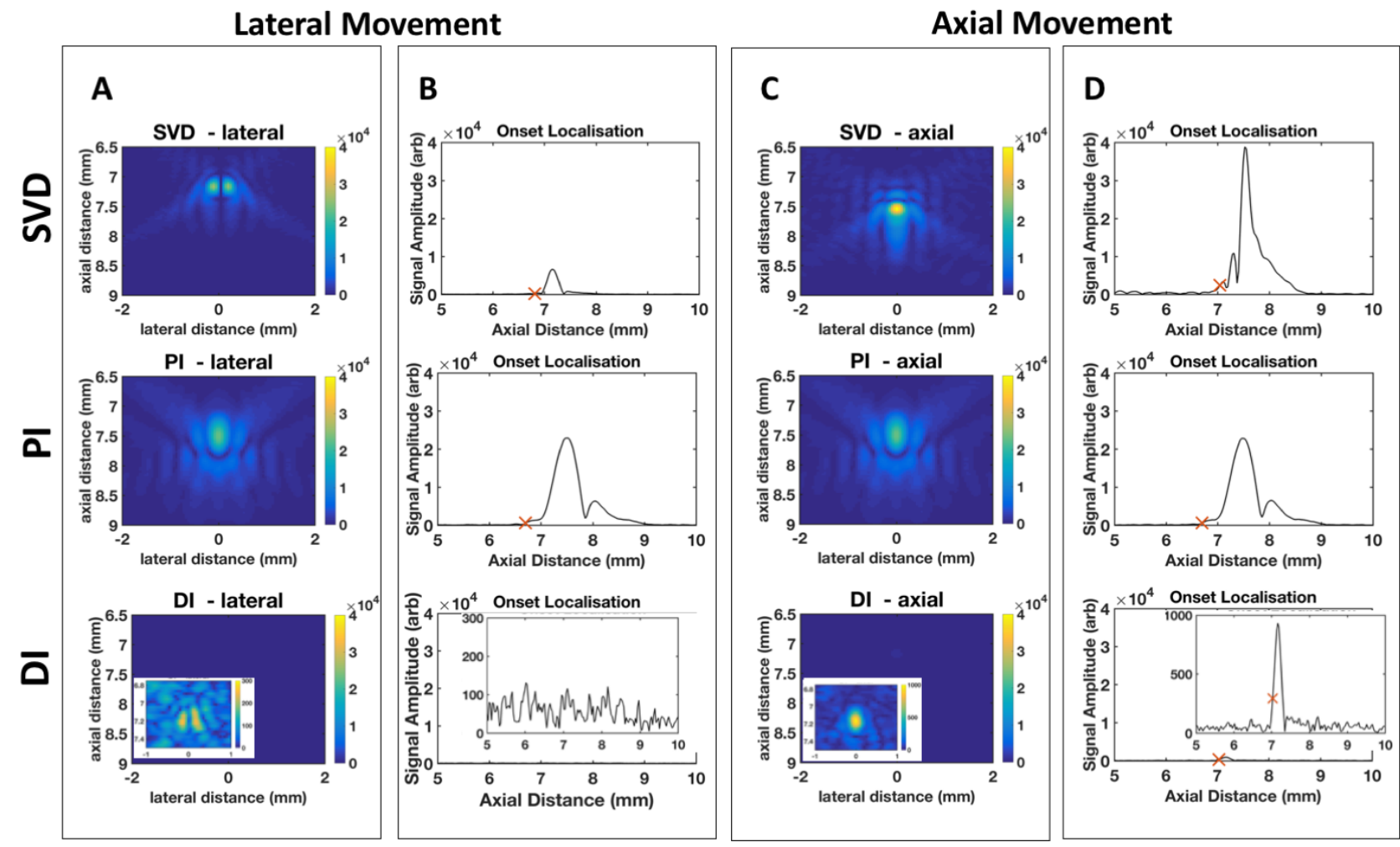

Fig. 14: Visualization of variation of MB point spread function. Shape of bubble signals for $0.5 \mathrm{~mm} / \mathrm{s}$ lateral (left) and axial (right) movement. PI was performed at a transmit frequency of $2 \mathrm{MHz}$ for PI (middle). A center transmit frequency of $6 \mathrm{MHz}$ was used for DI (bottom) and SVD (top). (A) The beamformed and envelope detected processed frames for SVD (top), PI (middle) and DI (bottom) for lateral flow. (B) The axial profile used for determining the MB axial position during the onset localization. The cross shows the localization position where possible. (C) Beamformed frames for axial flow. (D) Onset localization for axial movement. Insets are used for DI due to dynamic range used for overall comparison of the methods being too large.

missing segments (represented by a cross) and data points in Figure 13 (B). Figure 12 also shows that DI and SVD have lower CTR values for lateral motion.

The DI localizations are limited by noise. The results show that DI is only appropriate for MB speeds $>0.75 \mathrm{~mm} / \mathrm{s}$ or as flow becomes more axial.

Figure 13 shows that SVD can introduce bias on the order of hundreds of micrometres over a range of speeds and directions. PI is more suitable for applications with varying flow velocities as any bias is constant over the range of velocities investigated here.

- Applications with faster flow, with an axial component of flow velocity.

Figure 12 shows that the highest CTR for faster flow can be achieved using SVD processing. However, CTR values begin to plateau and even decrease by a few decibels for MBs with radii $>1 \mu \mathrm{m}$. This effect is due to the signal spreading more evenly over the singular value components with increasing speed as shown in Figure 12(C).

Figure 12 also shows that DI and SVD have higher CTR as the flow becomes more axial (90 degrees). For DI, at $0.5 \mathrm{~mm} / \mathrm{s}$, the localizations are only possible when there is some axial component of velocity. Although DI has a significantly lower CTR compared to PI and DI, the localization accuracy and precision are comparable. Thus, DI will still be a useful technique for faster blood flow or flow with some axial component.
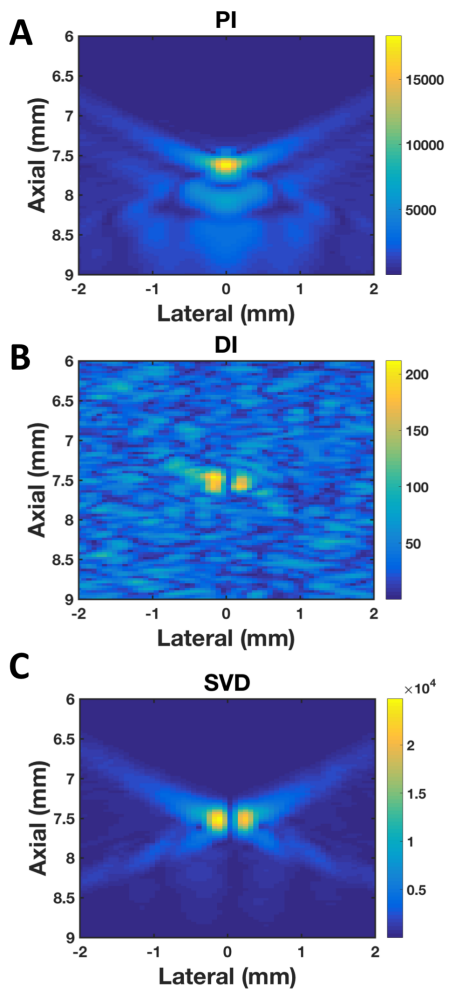

Fig. 15: Same data, different detection methods. Shape of MB signals for $0.5 \mathrm{~mm} / \mathrm{s}$ lateral flow at a center transmit frequency of $4 \mathrm{MHz}$ for PI (A), DI (B) and SVD (C). These images are from the same simulated data. 
Figure 12 (A and $\mathrm{B}$ ) show that there is more variation in CTR for PI across the different MB sizes compared to SVD and DI. This is shown by the relatively larger error bars for PI in Figure 13.

A degree of axial movement enables SVD to detect even the smallest MBs of $0.5 \mu \mathrm{m}$ radii. Axial precision falls to submicron for SVD localization of axial flow compared to $11.3 \mu \mathrm{m}$ for DI and $12.1 \mu \mathrm{m}$ for PI, see Figure 13 (D).

\section{PSF shape dependence}

Figure 14 shows how drastically different MB signals can appear using different methods. DI and SVD have signal loss in the center of the point spread functions. This signal loss is manifested in the bias introduced for lateral and axial accuracy shown in Figure 13(D) for SVD at 0 degrees. This only occurs for lateral motion. It is hypothesized that this is due to less variation between adjacent frames for a MB moving laterally compared to axially. Lower curvature at the center of the MB point spread function means that this signal is simply removed in DI filtering and decreased for SVD filtering.

This is even more striking in Figure 15 where the same frequency parameters are also used so that the filtering was applied on the same MB signals (note the different colour scales). Figure 15(A) for PI filtering shows significant ringing, whereas DI and SVD again have signal loss in the center of the point spread function.

\section{DiscusSION}

This work has investigated some of the factors affecting which detection method is most appropriate to extract the MB signal for super-resolution US imaging. Song et al. (2018) recently highlighted the need for a systematic review of $\mathrm{MB}$ extraction techniques, when discussing their use of DI [21]. Due to the challenges of creating a microvasculature phantom for experimental validation, simulation has been used here to provide a ground truth validation.

The results have shown that stack size in SVD influences the CTR of MB signals by changing the spread of the signal across the singular values. Thus, larger stack sizes do not always correspond to larger CTR values even when the eigenvalue cutoffs are adjusted. This is because some MB signal can be removed with tissue. SVD splits the signal into orthogonal components which cannot be simply split into tissue, MB and noise signal. Previous work with low frame rates has observed the overlap between $\mathrm{MB}$ and tissue signal [53]. This is especially problematic if the contrast in the unfiltered images is originally strong because SVD requires the assumption that tissue is significantly more energetic than noise and MB signal [54].

The results show that PI is more effective at lower frequencies. This is due to the resonant behaviour of MBs. For frequencies less than $3 \mathrm{MHz}$, using a probe with sufficiently wide bandwidth, it has the highest CTR. SVD CTR is less affected by frequency. This is true both for the investigations with and without incorporation of bandpass filtering. When incorporating the probe bandpass the detection techniques are affected both by the MB dependence on frequency, attenuation and nonlinear propagation in tissue, and the sensitivity of the available probes. The LA332 probe has a flatter frequency response than that of the PA230 probe. Thus SVD, already relatively robust to varying frequency without bandpass filtering, is a feasible choice over a wider range of frequencies for the LA332 probe modelled here. In comparison, transmit frequency choice for PI is more affected by the dependence of the $\mathrm{MB}$ response on frequency and by the more restrictive sensitivity of the PA230 probe. Although two specific probe examples have been simulated here, we suggest that these findings are also generally applicable when choices of detection method are being made based on available equipment. At the MI and pulse length used here, only a slight resonance behaviour can be observed in the results. At larger MI, MBs will exhibit more non-linear behaviour, including stronger subharmonics and ultraharmonics [55]. It should be noted that the non-linear behaviour of the MBs also depends on pulse length. Short pulses, more traditionally used for imaging studies, have been used here. But increasing the pulse length would generate higher CTR for both techniques and enhance MB non-linearity. This will be investigated in future work.

The results have shown that SVD and DI are more affected by speed and direction of MB flow than PI. SVD and DI do not work for stationary $\mathrm{MBs}$ and are less effective for lateral flow compared to axial. Variation in accuracy (bias) introduced over a physiological variable will cause the morphology of the imaged vessel to be distorted relative to the ground truth. Although PI does introduce an axial bias, this depends on the threshold chosen for the onset localization method. Constant bias over blood flow speed and direction will not affect the structural or functional information acquired.

Perhaps of particular concern for super-resolution is when filtering choices can change the shape of individual point spread functions. This can introduce errors such as in Figures 14 and 15 for SVD and DI where a single point spread function can resemble two adjacent signals. In addition, the potential for ringing or otherwise non-uniform axial profiles should be considered when choosing the localization method. This may be more problematic when using localization methods such as centroid and local maximum compared to onset.

Speed dependence will be influenced by choice of PRF. The CTR of slower MBs could be increased by decreasing the frame rate for the linear techniques. However, it is important to understand how this may affect the localizations of the faster MBs. Without a ground truth measurement of the MB speed it will be challenging to correct for any localization bias introduced. It is unnecessary to obtain a zero bias, but the bias should be constant over the flow directions and MB sizes, so the image is not blurred but only shifted. However, for fast axial flow, SVD is associated with high precision compared to PI. Thus, for some applications the improved precision may be considered more beneficial than a constant bias.

The computational cost of the three techniques is a further consideration that deserves discussion. SVD processing is significantly computationally more expensive that the simple techniques of PI and DI. This will have implications on the realtime applications of the techniques. Demene et al., (2015) reports that the computation times are of the order of a few seconds for typical data sizes and computational resources [22]. By minimizing the stack size, Desailly et al. (2016) reports 
satisfying CTR for a stack size of 30 frames, requiring a processing time of $<30 \mathrm{~ms}$ [24]. To the best of our knowledge, achieving the highest CTR possible with SVD in real time using larger stack sizes has not yet been demonstrated. In comparison, contrast pulse sequences have been widely implemented in real time [56].

There are limitations to this study. This work has attempted to give both a physically fair comparison of the techniques at different frequencies before moving to more practical scenarios with realistic transducer models. However, this is still an incomplete exploration of the huge parameter space, and so will not be applicable to every scenario.

One limitation of our study is the scope of the detection methods investigated. For example, there are other non-linear detection methods which have not been considered here [57, 58]. There is also the possibility of combining detection methods, for example, Harput et al. (2018) filters contrast enhanced ultrasound images with SVD [59]. However, it should be noted that results of this investigation would still be valid and combining these methods would result in an unknown combination of the errors associated with each technique.

In practice, each technique must be robust over a wide range of initial CTR and SNR levels. Tissue geometry, as shown in Figure 3(D-C), was kept constant across the simulations in this work. Physiologically relevant tissue acoustic properties and geometry were chosen to mimic a realistic tissue scenario. Simulations were performed at a relatively shallow depth of 7 $\mathrm{mm}$ to perform $\mathrm{k}$-Wave simulations in a reasonable time. This meant that a significant proportion of MB signal could be contained in the first singular vectors of the SVD decomposition. Noise was also kept constant across the simulations. However, in practice, SNR affects the efficacy of each technique and additional signal processing techniques may be required to improve image quality [60]. Modelling across a range of different tissue geometries, initial CTR values and SNR values is a topic for future investigation.

The MB motion considered here has been very simplistic and the tissue has been kept stationary. A key limitation of this study is that the effect of tissue motion has not been investigated. Demene et al. (2015) emphasizes the superior contrast to noise ratio achievable by SVD compared to traditional temporal filtering techniques [22]. However, a fair comparison of these techniques in the presence of motion is not trivial. In previous literature, motion has been limited experimentally by using a stereotactic frame [12] or rejection of frames with significant movement $[29,61]$. Future work will investigate how small tissue movements likely to remain, for example due to vessel pulsatility, would affect each MB detection method. For performing SR on data with more significant movement, post processing motion detection methods have been applied [62, 59]. Harput et al. (2018) recently applied a two-stage motion correction approach for non-rigid and affine motion to improve super-resolution imaging [59]. Here motion estimation was performed on Bmode images and then used to remove the motion from contrast enhanced images before the localization of isolated MBs. Hingot et al. (2018) used SVD twice; first to separate the strongest tissue components which can be used to make an estimation of the motion, and second (with different thresholding) to detect MBs [62]. The localizations were corrected following the MB detection using the tissue motion estimate. The order in which MB detection techniques and motion correction methods should be applied is a topic for future research. Simulations have suggested that motion correction techniques on B-mode data can be associated with errors on the order of tens of microns [59], which is comparable to the SR precisions reported in this work. Moreover, there are many types of motion to consider, from the periodic respiratory motion, to more unpredictable movements such as muscle spasms and probe motion. To comprehensively compare how motion will influence the choice of detection method different types of motion should be simulated followed by an appropriate motion correction technique with its associated error. This will need to be investigated in future work.

\section{CONCLUSION}

Overall, this work has begun the process of understanding how signal detection techniques impact the final superresolution image. This work has shown that the choice of detection technique is not trivial. Instead, it can dramatically change the shape and intensity of extracted signals and the resulting localization positions.

Localizations using the signals extracted using SVD and DI are dependent on the speed and direction of the blood flow being imaged. Under slow flow physiological conditions DI has too low a CTR and localization precision to be useful. This work suggests that PI is a more appropriate technique for accurately imaging the microvasculature flow simulated here. PI is most effective at center transmit frequencies $\leq 3 \mathrm{MHz}-$ assuming a probe with sufficiently high bandwidth is available. SVD localization had improved precisions at higher frequencies, where results showed that SVD performed on data acquired at a higher transmit frequency of $6 \mathrm{MHz}$ would provide better overall CTR and precision than PI performed at $2 \mathrm{MHz}$. However, the variation in accuracy for SVD over the speed range studied here will cause distortion of the structural information. In particular this may affect the visualization of regions where there are a range of difference vessel sizes and flow rates. For example, branching vasculature such as that from arterioles to capillaries may be misrepresented.

Overall, this velocity dependence of DI and SVD mean that they are less suitable for visualization of tumor microvasculature than PI.

\section{REFERENCES}

1. Foster, F.S., et al., Ultrasound for the visualization and quantification of tumor microcirculation. Cancer and Metastasis reviews, 2000. 19(1-2): p. 131-138.

2. Goertz, D.E., et al., High-frequency color flow imaging of the microcirculation. Ultrasound in medicine \& biology, 2000. 26(1): p. 63-71.

3. Cosgrove, D., Ultrasound contrast agents: an overview. European journal of radiology, 2006. 60(3): p. 324-330.

4. Blomley, M.J., et al., Microbubble contrast agents: a new era in ultrasound. British Medical Journal, 2001. 322(7296): p. 1222.

5. Gessner, R.C., et al., Acoustic angiography: a new imaging modality for assessing microvasculature architecture. Journal of Biomedical Imaging, 2013. 2013: p. 14. 
6. Dayton, P.A., et al., The implementation of acoustic angiography for microvascular and angiogenesis imaging. Conference proceedings : .. 2014. Annual International Conference of the IEEE Engineering in Medicine and Biology Society. IEEE Engineering in Medicine and Biology Society. 2014: p. 4283-4285.

7. Shelton, S.E., et al., Quantification of microvascular tortuosity during tumor evolution using acoustic angiography. Ultrasound in Medicine and Biology, 2015. 41(7): p. 1896-1904.

8. Viessmann, O., et al., Acoustic super-resolution with ultrasound and microbubbles. Physics in medicine and biology, 2013. 58(18): p. 6447.

9. O'Reilly, M.A. and K. Hynynen, A super-resolution ultrasound method for brain vascular mapping. Medical physics, 2013.40(11): p. 110701.

10. Desailly, Y., et al., Sono-activated ultrasound localization microscopy. Applied Physics Letters, 2013. 103(17): p. 174107.

11. Christensen-Jeffries, K., et al., In vivo acoustic superresolution and super-resolved velocity mapping using microbubbles. Medical Imaging, IEEE Transactions on, 2015. 34(2): p. 433-440.

12. Errico, C., et al., Ultrafast ultrasound localization microscopy for deep super-resolution vascular imaging. Nature, 2015. 527(7579): p. 499-502.

13. Siepmann, M., et al. Imaging tumor vascularity by tracing single microbubbles. in Ultrasonics Symposium (IUS), 2011 IEEE International. 2011. IEEE.

14. Couture, O., et al. Microbubble ultrasound superlocalization imaging (MUSLI). in Ultrasonics Symposium (IUS), 2011 IEEE International. 2011. IEEE.

15. Bar-Zion, A., et al., Fast vascular ultrasound imaging with enhanced spatial resolution and background rejection. IEEE transactions on medical imaging, 2017. 36(1): p. 169-180.

16. Bar-Zion, A., et al., SUSHI: Sparsity-based Ultrasound Super-resolution Hemodynamic Imaging. IEEE transactions on ultrasonics, ferroelectrics, and frequency control, 2018.

17. Desailly, Y., et al., Resolution limits of ultrafast ultrasound localization microscopy. Physics in medicine and biology, 2015. 60(22): p. 8723.

18. Simpson, D.H., C.T. Chin, and P.N. Burns, Pulse inversion Doppler: a new method for detecting nonlinear echoes from microbubble contrast agents. IEEE transactions on ultrasonics, ferroelectrics, and frequency control, 1999. 46(2): p. 372-382.

19. Dencks, S., et al. Determination of adequate measurement times for super-resolution characterization of tumor vascularization. in Ultrasonics Symposium (IUS), 2017 IEEE International. 2017. IEEE.

20. Lin, F., et al., Optimizing Sensitivity of Ultrasound Contrast-Enhanced Super-Resolution Imaging by Tailoring Size Distribution of Microbubble Contrast Agent. Ultrasound in Medicine and Biology, 2017. 43(10): p. 2488-2493.

21. Song, P., et al., Improved Super-Resolution Ultrasound Microvessel Imaging With
Spatiotemporal Nonlocal Means Filtering and Bipartite Graph-Based Microbubble Tracking. IEEE transactions on ultrasonics, ferroelectrics, and frequency control, 2018. 65(2): p. 149-167.

22. Demené, C., et al., Spatiotemporal clutter filtering of ultrafast ultrasound data highly increases Doppler and fUltrasound sensitivity. IEEE transactions on medical imaging, 2015. 34(11): p. 2271-2285.

23. Song, P., et al., Ultrasound Small Vessel Imaging With Block-Wise Adaptive Local Clutter Filtering. IEEE Transactions on Medical Imaging, 2017. 36(1): p. 251-262.

24. Desailly, Y., et al., Contrast enhanced ultrasound by real-time spatiotemporal filtering of ultrafast images. Physics in medicine and biology, 2016. 62(1): p. 31.

25. Baranger, J., et al., Adaptive spatiotemporal SVD clutter filtering for Ultrafast Doppler Imaging using similarity of spatial singular vectors. IEEE Transactions on Medical Imaging, 2018.

26. Van der Meer, S., et al. The resonance frequency of SonoVue ${ }^{\mathrm{TM}}$ as observed by high-speed optical imaging. in Ultrasonics Symposium, 2004 IEEE. 2004. IEEE.

27. Tang, M.-X., N. Kamiyama, and R.J. Eckersley, Effects of nonlinear propagation in ultrasound contrast agent imaging. Ultrasound in Medicine and Biology, 2010. 36(3): p. 459-466.

28. Lin, F., J.D. Rojas, and P.A. Dayton. Super resolution contrast ultrasound imaging: Analysis of imaging resolution and application to imaging tumor angiogenesis. in Ultrasonics Symposium (IUS), 2016 IEEE International. 2016. IEEE.

29. Lin, F., et al., 3-D ultrasound localization microscopy for identifying microvascular morphology features of tumor angiogenesis at a resolution beyond the diffraction limit of conventional ultrasound. Theranostics, 2017. 7(1): p. 196.

30. Yuan, F., et al., Vascular permeability and microcirculation of gliomas and mammary carcinomas transplanted in rat and mouse cranial windows. Cancer research, 1994. 54(17): p. 45644568.

31. McDonald, D.M. and P.L. Choyke, Imaging of angiogenesis: from microscope to clinic. Nature medicine, 2003. 9(6): p. 713-725.

32. Fukumura, D., et al., Tumor Microvasculature and Microenvironment: Novel Insights Through Intravital Imaging in Pre-Clinical Models. Microcirculation, 2010. 17(3): p. 206-225.

33. Korn, C. and H.G. Augustin, Mechanisms of vessel pruning and regression. Developmental cell, 2015. 34(1): p. 5-17.

34. Treeby, B.E., et al., Modeling nonlinear ultrasound propagation in heterogeneous media with power law absorption using ak-space pseudospectral method. The Journal of the Acoustical Society of America, 2012. 131(6): p. 4324-4336.

35. Cox, B.T., et al. Experimental validation of photoacoustic $k$-space propagation models. in Biomedical Optics 2004. 2004. International Society for Optics and Photonics. 
36. Tabei, M., T.D. Mast, and R.C. Waag, A k-space method for coupled first-order acoustic propagation equations. The Journal of the Acoustical Society of America, 2002. 111(1): p. 53-63.

37. Azhari, H., Basics of biomedical ultrasound for engineers. 2010: John Wiley \& Sons.

38. Mamou, J. and M.L. Oelze, Quantitative ultrasound in soft tissues. 2013: Springer.

39. Boni, E., et al. Multi-channel raw-data acquisition for ultrasound research. in Digital System Design (DSD), 2014 17th Euromicro Conference on. 2014. IEEE.

40. Doinikov, A.A. and A. Bouakaz, Review of shell models for contrast agent microbubbles. IEEE transactions on ultrasonics, ferroelectrics, and frequency control, 2011. 58(5): p. 981-993.

41. Faez, T., et al., 20 years of ultrasound contrast agent modeling. IEEE transactions on ultrasonics, ferroelectrics, and frequency control, 2013. 60(1).

42. Marmottant, P., et al., A model for large amplitude oscillations of coated bubbles accounting for buckling and rupture. The Journal of the Acoustical Society of America, 2005. 118(6): p. 3499-3505.

43. Sijl, J., et al., "Compression-only" behavior: A second-order nonlinear response of ultrasound contrast agent microbubbles. The Journal of the Acoustical Society of America, 2011. 129(4): p. 17291739 .

44. Gorce, J.-M., M. Arditi, and M. Schneider, Influence of bubble size distribution on the echogenicity of ultrasound contrast agents: a study of Sonovue . $^{\mathrm{TM}}$. Investigative radiology, 2000. 35(11): p. 661-671.

45. van der Meer, S.M., et al., Microbubble spectroscopy of ultrasound contrast agents. The Journal of the Acoustical Society of America, 2007. 121(1): p. 648656.

46. $\mathrm{Tu}, \mathrm{J}$., et al., Estimating the shell parameters of SonoVue ${ }^{\circledR}$ microbubbles using light scattering. The Journal of the Acoustical Society of America, 2009. 126(6): p. 2954-2962.

47. International Civil Aviation Organization, I.C.A., Manual of the ICAO Standard Atmosphere, Doc 7488CD. 1993.

48. Leighton, T., The acoustic bubble. 2012: Academic press.

49. Treeby, B., B. Cox, and J. Jaros, $k$-Wave: $A$ MATLAB toolbox for the time domain simulation of acoustic wave fields. 2012.

50. Montaldo, G., et al., Coherent plane-wave compounding for very high frame rate ultrasonography and transient elastography. IEEE transactions on ultrasonics, ferroelectrics, and frequency control, 2009. 56(3): p. 489-506.

51. Lindsey, B.D., S.E. Shelton, and P.A. Dayton, Optimization of contrast-to-tissue ratio through pulse windowing in dual-frequency "acoustic angiography" imaging. Ultrasound in medicine \& biology, 2015. 41(7): p. 1884-1895.

52. Christensen-Jeffries, K., et al., Microbubble Axial Localization Errors in Ultrasound Super-Resolution Imaging. IEEE transactions on ultrasonics, ferroelectrics, and frequency control, 2017. 64(11): p. 1644-1654.
53. Lediju, M.A., et al., A motion-based approach to abdominal clutter reduction. IEEE transactions on ultrasonics, ferroelectrics, and frequency control, 2009. 56(11): p. 2437-2449.

54. Mauldin, F.W., Jr., D. Lin, and J.A. Hossack, The singular value filter: a general filter design strategy for PCA-based signal separation in medical ultrasound imaging. IEEE Transactions on Medical Imaging, 2011. 30(11): p. 1951-64.

55. Shi, W.T. and F. Forsberg, Ultrasonic characterization of the nonlinear properties of contrast microbubbles. Ultrasound in medicine \& biology, 2000. 26(1): p. 93-104.

56. Porter, T.R. and F. Xie, Myocardial perfusion imaging with contrast ultrasound. JACC: Cardiovascular Imaging, 2010. 3(2): p. 176-187.

57. Phillips, P. Contrast pulse sequences (CPS): imaging nonlinear microbubbles. in Ultrasonics Symposium, 2001 IEEE. 2001. IEEE.

58. Eckersley, R.J., C.T. Chin, and P.N. Burns, Optimising phase and amplitude modulation schemes for imaging microbubble contrast agents at low acoustic power. Ultrasound in medicine \& biology, 2005. 31(2): p. 213-219.

59. Harput, S., et al., Two-Stage Motion Correction for Super-Resolution Ultrasound Imaging in Human Lower Limb. IEEE transactions on ultrasonics, ferroelectrics, and frequency control, 2018. 65(5): p. 803-814.

60. Bar-Zion, A.D., et al., Denoising of contrastenhanced ultrasound cine sequences based on a multiplicative model. IEEE Transactions on Biomedical Engineering, 2015. 62(8): p. 1969-1980.

61. Ackermann, D. and G. Schmitz, Detection and Tracking of Multiple Microbubbles in Ultrasound BMode Images. Ultrasonics, Ferroelectrics, and Frequency Control, IEEE Transactions on, 2016. 63(1): p. 72-82.

62. Hingot, V., et al., Subwavelength motion-correction for ultrafast ultrasound localization microscopy. Ultrasonics, 2017. 77: p. 17-21. 